\title{
Triple-pomeron amplitude in the effective action approach
}

\author{
M. A. Braun ${ }^{\text {a }}$ \\ Saint Petersburg State University, Saint Petersburg, Russia
}

Received: 16 June 2020 / Accepted: 13 August 2020 / Published online: 25 August 2020

(C) The Author(s) 2020

\begin{abstract}
In the effective action approach the imaginary part of the triple pomeron amplitude is calculated. The found dependence on the longitudinal momentum transfer $e_{-}$is found to separate as a simple factor $1 /\left|e_{-}\right|$. This result is used to calculate the high-mass diffraction on a hadron and double scattering cross-section off a composite target.
\end{abstract}

\section{Motivation}

Rather long ago in the study of interaction of a colorless projectile with two colorless targets the triple-pomeron vertex $\Gamma$ was constructed both in the BFKL approach $[1,2]$ and dipole picture [3-5]. The simplest scattering amplitudes involving the triple pomeron vertex include the diffractive scattering on the hadron Fig. 1 and the double scattering on the deuteron (or nucleus) Fig. 2.

Consider first the diffractive scattering Fig. 1. We denote the scattering amplitude as $H$, the momentum of the projectile in the c.m. system as $k$ with $k_{-}=k_{\perp}=0$ and the initial and final momenta of each of the target as $l$ and $l^{\prime}$ With $l_{+}=l_{\perp}=0$ and $l^{\prime}-l=e$. The diffractive mass squared is $M^{2}=\left(k+l-l^{\prime}\right)^{2}=-2 k_{+} e_{-}$and we assume $s>>M^{2}>>l_{\perp}^{2}$ The diffractive cross-section is given by

$d \sigma=\frac{d e_{-} d^{2} l_{\perp}^{\prime}}{16 \pi^{3} l_{-}} \frac{1}{s} \frac{1}{2 i} \operatorname{Disc}_{d} \mathcal{A}(e)$,

where $\operatorname{Disc}_{d} \mathcal{A}$ is a particular, diffractive discontinuity of the amplitude on the cut passing between the two target in Fig. 1. Using the relation between $e_{-}$and the diffractive mass we can rewrite (1) as

$d \sigma=\frac{d M^{2} d^{2} l_{\perp}^{\prime}}{16 \pi^{3} s^{2}} \frac{1}{2 i} \operatorname{Disc}_{d} \mathcal{A}(e)$.

a e-mail: braun1@ pobox.spbu.ru (corresponding author)
It is convenient to separate the trivial energetic factor and define

$\frac{1}{i} \operatorname{Disc}_{d} \mathcal{A}=\frac{2 s^{2}}{k_{+}} H$.

In terms of amplitude $H$ we find

$d \sigma=\frac{d M^{2} d^{2} l_{\perp}^{\prime}}{16 \pi^{3} k_{+}} H(e)=\frac{d M^{2} d^{2} l_{\perp}^{\prime}}{8 \pi^{3} M^{2}}\left|e_{-}\right| H(e)$.

Now consider the double scattering Fig. 2. The standard treatment (see e.g. [6]) takes into account that in the rest system the spatial components of the nucleons in the deuteron or nucleus are small and the energy component is still smaller. Because of this the high-energy part $\mathcal{A}$ does not depend on the transversal components but does depend on the $z$-component. Integrating out the transversal components one separates a factor carrying information on the composite target but the integration over the $z$-component remains. Boosted to the c.m. system the latter transforms into the "-" component $e_{-}$. So if one denotes $D$ the whole diagram in Fig. 2 one obtains the cross-section for the double scattering on the deuteron as

$\sigma_{d}=\frac{1}{S} \operatorname{Im} D=\frac{1}{2 \pi} \int d q_{z} \operatorname{Im} \mathcal{A}\left(q_{z}\right) e^{-i q_{z} z}\left|\psi_{d}\left(r_{\perp}=0, z\right)\right|^{2}$.

([6], eq. (90) in that paper with slightly different notations). Here $m$ is the nucleon mass. Passing to the c.m. system one has $q_{z}=e_{-} m / k_{+}$. At large $k_{+}$one can neglect the exponential in (5) and gets

$\sigma_{d}=\frac{1}{4 \pi^{2} k_{+} s}\left\langle\frac{1}{r^{2}}\right\rangle_{d} \int d e_{-} \operatorname{Im} \mathcal{A}\left(e_{-}\right)$,

where this time $e_{\perp}=0$. The same derivation for the nucleus target gives the double cross-section on the nucleus at fixed impact parameter $b$

$\frac{d \sigma_{A}}{d^{2} b}=\frac{A(A-1)}{4 \pi k_{+} s} T^{2}(b) \int d e_{-} \operatorname{Im} \mathcal{A}(e)$. 


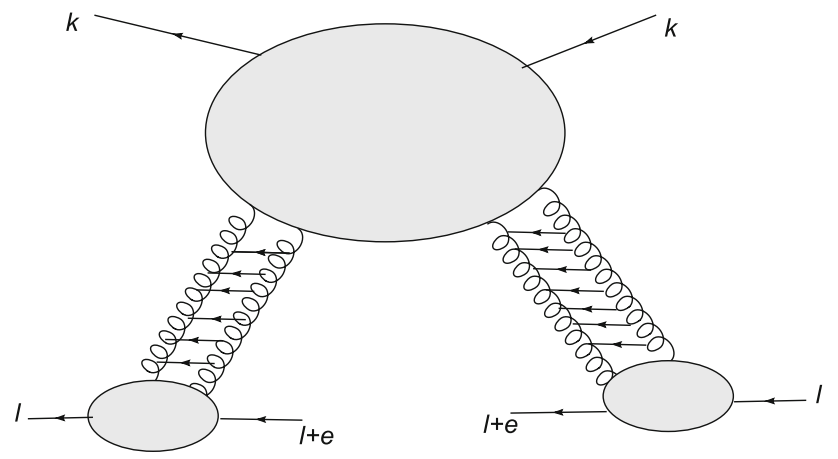

Fig. 1 Diffractive scattering off a hadron

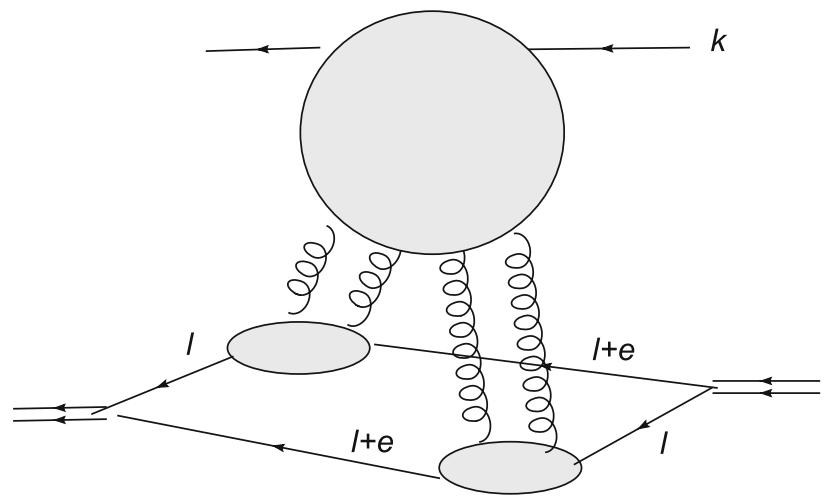

Fig. 2 Double scattering on the deuteron or nucleus. In the second case the spectator nucleons are not shown

As opposed to the diffractive cross-section (2), for the double scattering one has to take the total imaginary part of $\mathcal{A}$. However it is trivially related to its diffractive part by the famous AGK rules $[7,8]$. They dictate that the relation between the discontinuities corresponding to zero (diffractive), one and two cut pomerons are related as 1:2:-4. Their sum gives the total discontinuity, which results equal to the diffractive one with the opposite sign:

$\operatorname{Im} \mathcal{A}(e)=-\frac{1}{2 i} \operatorname{Disc}_{d} \mathcal{A}(e)$.

Relating $\mathcal{A}$ and $H$ by (3) we find finally for the nucleus

$\frac{d \sigma_{A}}{d^{2} b}=-\frac{A(A-1)}{2 \pi} T^{2}(b) \int d e_{-} H\left(e_{-}\right)$

and for the deuteron

$\sigma_{d}=-\frac{1}{\pi^{2}}\left\langle\frac{1}{r^{2}}\right\rangle_{d} \int d e_{-} H\left(e_{-}\right)$.

Inspecting expressions for the cross-sections for both cases one arrives at the following conclusions.

First it is sufficient to know the diffractive cross-sections. The cross-sections for the double scattering can be found from the AGK relation (8) after attaching the necessary factor for the compound target.

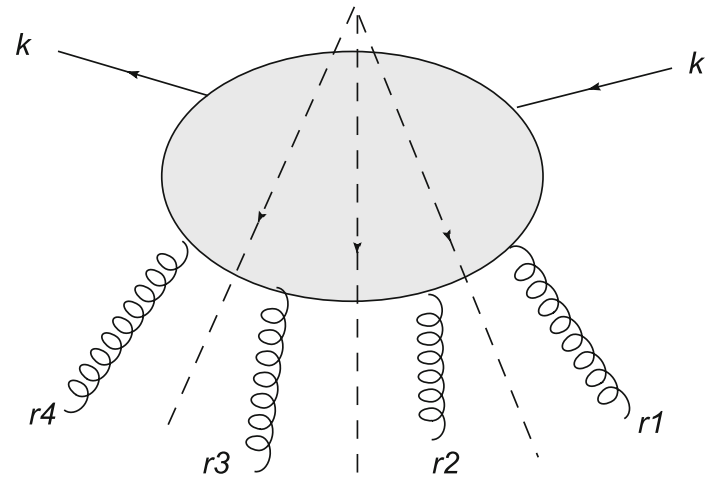

Fig. 3 Triple cut of the triple-pomeron amplitude

Second one has to know the dependence of the amplitude $H$ on the longitudinal momentum transfer $e_{-}$. This can be done only if one retains the dependence on longitudinal variable in the calculation of the relevant diagrams. In the old derivations one usually studied the diagrams with free outgoing reggeons and calculated the triple discontinuity on the three cuts passing between them Fig. 3. After that one integrated over the three "-" components of these reggeons to obtain a purely transversal expression (see e.g. $[1,9,10]$ ) Passage to the final pomerons was then made directly in the transverse space. The $e_{-}$dependence was traded for the $M^{2}$ dependence corresponding to the rapidity of the incoming reggeon. It is not convenient for the double scattering where one has to explicitly integrate over all values of $e_{-}$.

The clear way to directly produce the desired dependence is to use Lipatov's effective action approach (LEA), which generates amplitudes with full dependence on the longitudinal variables. This motivates our study, in which we calculate the amplitude $\mathcal{A}(e)$ using the effective action. In fact the amplitude $\mathcal{A}$ itself is most complicated and has to be calculated in the general gauge due to the fact that all intermediate gluons initially lie off the mass shall. However the amplitude $\mathcal{A}$ as a whole appears only in the expression for the elastic scattering on the composite target where one should know $|\mathcal{A}|^{2}$ at the momentum transfer different from zero. Leaving this task for future calculations we restrict here to the discussed physical cases where one only needs the diffractive imaginary part of $\mathcal{A}$. Apart from drastically reducing the number of relevant diagrams it allows to work in the light-cone gauge for the intermediate real gluon taking its polarization vector $\epsilon$ orthogonal to $l$, that is with $\epsilon_{+}=0$.

Note that the $e_{-}$- dependence can of course be obtained also from the triple discontinuity. To do this one has to return to the 4 dimensional picture and then establish the relation between the full, diffractive and triple discontinuities, more or less along the line of [8]. The effective action is just a convenient instrument for handling the diagrams with reggeized gluons but by itself does not introduce any new contributions. It only allows to obtain the result in a more direct and eco- 
nomic way, especially since the main building blocks have been earlier found in this approach.

To see the $e_{-}$dependence it is sufficient to use the perturbative approach and start with the lowest order. This means that we can approximate all pomerons with the double gluon exchange. Also one can take simple $q \bar{q}$ loops for the participants. Our calculations are divided in parts corresponding to the number of incoming reggeons attached to the projectile 2 , 3 and 4 and studied in the next Sects. 2, 3 and 4, respectively. Having in mind the applications either to the diffractive scattering or double scattering on the nucleus, we consider the case when the incoming pomeron is the forward one but the two outgoing ones are generally non-forward.

\subsection{The relevant vertices in the effective action}

The LEA approach was presented in the detail in $[11,12]$ and the following Fenian rules were formulated in [13]. Here we only briefly discuss its main points to make the following derivation. In the LEA within a rapidity slice of finite dimension gluons are described by the usual (matrix) gluon field $G=-i t^{a} G^{a}$. The two reggeon field $R_{ \pm}$with the only non-zero longitudinal components connect slices with widely different rancidities. The effective Lagrangian describes the interaction of gluons and reggeons with a given rapidity slice.It takes the form $[11,12]$ :

$$
\begin{aligned}
& \mathcal{L}_{\text {eff }}=\mathcal{L}_{Q C D}(V+G) \\
& \quad+2 \operatorname{Tr}\left\{\left(j_{+}(G+R)-R\right) \partial_{\perp}^{2} G_{-}\right. \\
& \left.\left.\quad+\left(j_{-}(G+R)-R\right) \partial_{\perp}^{2} G_{+}\right\}\right),
\end{aligned}
$$

where $\mathcal{L}_{Q C D}(V)$ is the usual QCD Lagrangian and

$j_{ \pm}(G)=\sum_{n=0}^{\infty}(-g)^{n} G_{ \pm}\left(\partial_{ \pm}^{-1} G_{ \pm}\right)^{n}$.

The shift $G \rightarrow G+R$ with $R_{\perp}=0$ is done to exclude direct gluon-reggeon transitions. The reggeon propagator in momentum representation is

$\Delta^{a b}\left(y^{\prime}-y, q\right)=<R_{+}^{a}\left(y^{\prime}\right) R_{-}^{b}(y)>=-i \frac{\delta_{a b}}{q_{\perp}^{2}} \theta\left(y^{\prime}-y\right)$.

Here $a, b$ are color indices. It couples field $R_{-}$interacting with a group of a higher rapidity $y^{\prime}$ and field $R_{+}$interacting with a group of a smaller rapidity $y$. From the kinematical constraints it follows that

$\partial_{\mp} R_{ \pm}=0$.

The effective Lagrangian generates elementary vertices for the interaction of gluons and reggeons which come both from the QCD part and the rest "induced" part and so separate in basic and induced vertices. In higher orders propagation of

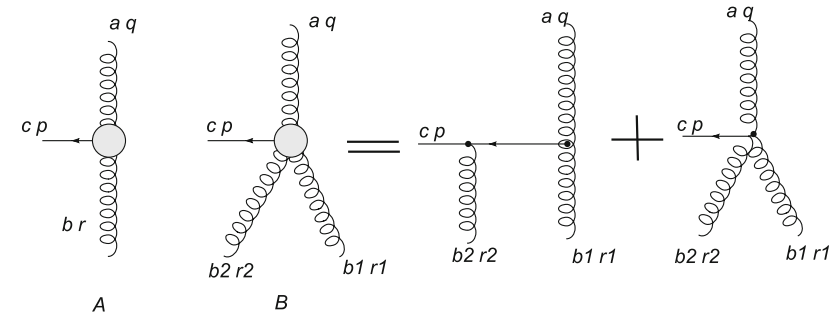

Fig. 4 Vertexes $\Gamma_{R \rightarrow G+R}$ (a) and $\Gamma_{R \rightarrow G+2 R}$ (b). The second vertex should be symmetrized in the outgoing reggeons

intermediate virtual gluons gives rise to more complicated compound vertices, which contain one or several off-shell intermediate gluon states.

In our calculations it will be sufficient to know two basic vertices for gluon production in the interaction of one incoming reggeon with one outgoing ("the Lipatov vertex") $\Gamma_{R \rightarrow G+R}$ or two outgoing reggeons $\Gamma_{R \rightarrow G+2 R}$. The first vertex has been known since long ago (see [14-16]), the second was calculated in LEA in $[17,18]$. We reproduce them here in the light-cone gauge with respect to the target with the gluon polarization vector $\epsilon$ orthogonal to the incoming momentum of the target $l:(l \epsilon)=0$, so that $\epsilon_{+}=0$.

In this gauge the Lipatov vertex is (Fig. 4a)

$$
\begin{aligned}
& \Gamma_{R \rightarrow G+R}=g q^{2} L(p, r), \\
& L(p, q)=\frac{(p e)_{\perp}}{p_{\perp}^{2}}-\frac{(p+r, e)_{\perp}}{(p+r)_{\perp}^{2}} .
\end{aligned}
$$

Vertex $\Gamma_{R \rightarrow G+2 R}$ is given by the sum ( Fig. 4b)

$\Gamma_{R \rightarrow G+2 R}=W_{1}+R_{1}+(1 \leftrightarrow 2)$.

Here vertex $R_{1}$ is

$R_{1}=i g^{2} \frac{q_{\perp}^{2}}{r_{1-}} f^{a b_{1} d} f^{d b_{2} c} L\left(p, r_{2}\right)$.

Vertex $W_{1}$ is

$W_{1}=-i g^{2} \frac{2 q_{+} q_{\perp}^{2}}{\left(q-r_{1}\right)^{2}+i 0} f^{a b_{1} d} f^{d b_{2} c} B\left(p, r_{2}, r_{1}\right)$

where the "Bartels vertex" $B$ is

$B\left(p, r_{2}, r_{1}\right)=L\left(p+r_{2}, r_{1}\right)$.

$(1 \leftrightarrow 2)$ means the interchange of the two outgoing reggeons. Note that $R_{1}$ contains a singularity at $r_{1-}=0$, which should be understood in the principal value prescription.

\section{Two reggeons attached to the projectile}

The diagram corresponding to this amplitude is shown in Fig. 5. For the diffractive contribution the cut should go between the two targets. 


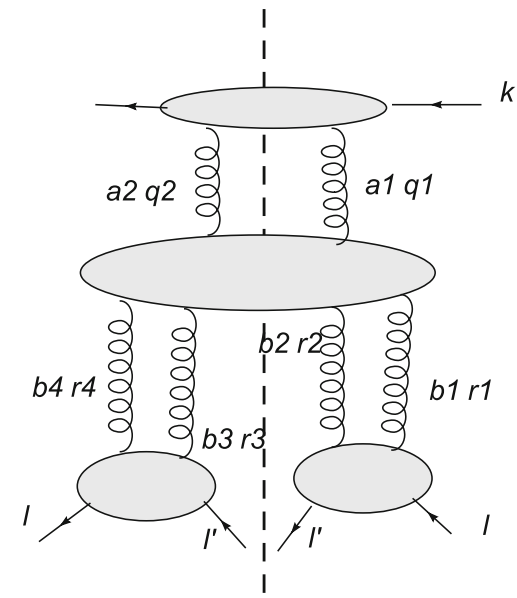

Fig. 5 The diffractive amplitude with two reggeons attached to the projectile

In the lowest order the blobs are usually taken as $q \bar{q}$ loops with the two reggeons attached to the quarks and antiquarks in all different ways. The reggeons are just gluons with the propagators depending on only their transverse momenta. The projectile impact factor depends on $q_{1-}$. The target impact factors depend on " + " components of $r$ 's. The central blob is the vertex $\Gamma_{2 R \rightarrow 4 R}$ for the transition of two incoming reggeons into four outgoing ones. Apart from the transverse momenta it depends on $q_{1+}=-q_{2+}, r_{1-}$ and $r_{4-}$. One has $r_{1}+r_{2}=-r_{3}-r_{4}=e$ with $e_{+}=0$

Integration over $q_{-}, r_{1+}$ and $r_{4+}$ are factored out. Only the impact factors depend on them. So one can immediately do these integrations by using energetic variables $\left(k-q_{1}\right)^{2}$, $\left(l+r_{1}\right)^{2}$ and $\left(l+r_{4}\right)^{2}$ and rotating the Feynman integration contour around the cut of the impact factors. In this way one obtains the standard Impact functions $D^{(0)}$ depending only on the corresponding transverse momenta of the attached reggeons multiplied by the overall energetic factor $2 s^{2} / k_{+}$ entering (3). Factors $1 / 2 \pi$ associated with these longitudinal integrations are usually included into the integration volume $d \tau_{\perp}$ over the transverse momenta in which for each momentum $q$ appears $d^{2} q / 8 \pi^{3}$. Also since the singularities of the impact factors are supposed to lie at limited values the momenta, $q_{-}, r_{1+}$ and $r_{4+}$ become of the order $1 / k_{+}$that is practically zero at large energies. As a result the incoming reggeons acquire $q_{-}=0$ and the outgoing ones $r_{+}=0$ in accordance with the effective action.

In this way one obtains the diffractive amplitude $\mathrm{H}_{2} \mathrm{com}$ ing from the contributions with two reggeons attached to the projectile in the form of the integral

$$
\begin{gathered}
H_{2}(e)=\int d \tau_{\perp} P^{(0)}\left(q_{1}, q_{2}\right) P^{(0)}\left(r_{1}, r_{2}\right) P^{(0)}\left(r_{4}, r_{3}\right) \\
\int \frac{d q_{1+} d r_{1-} d r_{4-}}{(2 \pi)^{3}} \Gamma_{2 R \rightarrow 4 R}\left(q_{1+}, r_{1-}, r_{2-}\right) .
\end{gathered}
$$

Here $d \tau_{\perp}$ is the phase volume for the integration over the transverse momenta $q_{1}, r_{1}$ and $r_{4}$ and it is implied that the vertex $\Gamma_{2 R \rightarrow 4 R}$ also depends on these momenta.

The summation over colors gives $N^{4}$ where $N$ is the number of colors. We work in the approximation $N>>1$.

The integration over $q_{1+}$ is done due to the cut which provides $-2 \pi \delta\left(\left(q_{1}-e\right)^{2}\right)$. One gets factor $-i / 4\left|e_{-}\right|$and puts $q_{1+}=p_{\perp}^{2} / 2 e_{-}=-q_{2+}$. Note that $p_{-}=-e_{-}$has to be greater than zero, which automatically requires $e_{-}<0$. Otherwise the discontinuity is zero. With $e_{-}<0$ one has $q_{1+}>0$ and $q_{2+}<0$.

The rest part of $\Gamma_{2 R \rightarrow 4 R}\left(q_{1+}, r_{1-}, r_{2-}\right)$ is the product of two vertices $\Gamma_{R \rightarrow G+2 R}$ given by (16). Integration over $r_{1-}$ and $r_{4-}$ are separated. One gets

$$
\int \frac{d r_{1-}}{2 \pi} \Gamma_{R \rightarrow G+2 R}\left(r_{1}, r_{2} \mid q_{1}\right)=2 \int \frac{d r_{1-}}{2 \pi} W\left(r_{1}, r_{2} \mid q_{1}\right)
$$

because the interchange $(1 \leftrightarrow 2)$ does not change the result and integration over of $R$ gives zero due to the principal value prescription. We have

$$
\begin{aligned}
& \int \frac{d r_{1-}}{2 \pi} \frac{1}{\left(q_{1}-r_{1}\right)^{2}+i 0} \\
& =\int \frac{d r_{1-}}{2 \pi} \frac{1}{-2 q_{1+} r_{1-}+\left(q_{1}-r_{1}\right)_{\perp}^{2}+i 0}=-i \frac{1}{4\left|q_{1+}\right|},
\end{aligned}
$$

so that after integration we get

$\int \frac{d r_{1-}}{2 \pi} W\left(r_{1}, r_{2} \mid q_{1}\right)=-g^{2} \frac{q_{1+} q_{1}^{2}}{2\left|q_{1+}\right|} B\left(p, r_{2}, r_{1}\right)$,

where $p=q_{1}-e$. Integration of the second vertex $W$ gives in the same way

$\int \frac{d r_{4-}}{2 \pi} W\left(r_{4}, r_{3} \mid q_{2}\right)=-g^{2} \frac{q_{2+} q_{2}^{2}}{2\left|q_{2+}\right|} B\left(-p, r_{3}, r_{4}\right)$.

Conjugation requires to invert all vectors but this does not change the $q_{2+} B\left(-p, r_{3}, r_{4}\right)$. The emerging factor $q_{1+} q_{2+} /\left|q_{1+}\right|\left|q_{2+}\right|=-1$.

Collecting all factors we find

$$
\begin{aligned}
& H_{2}(e) \\
& =\frac{1}{2\left|e_{-}\right|} g^{4} N^{4} \int d \tau_{\perp} D^{(0)}\left(q_{1}, q_{2}\right) P^{(0)}\left(r_{1}, r_{2}\right) P^{(0)}\left(r_{4}, r_{3}\right) \\
& \quad \times\left(\frac{p+r_{2}}{\left(p+r_{2}\right)^{2}}-\frac{q_{1}}{q_{1}^{2}}\right)\left(\frac{-p+r_{3}}{\left(-p+r_{3}\right)^{2}}-\frac{q_{2}}{q_{2}^{2}}\right)
\end{aligned}
$$

where one can consider all vectors to be Euclidean 2dimensional. The product of two vectors which appears is in fact the well-known Bartels kernel for transition of two reggeons into four

$$
K_{2 \rightarrow 4}\left(r_{4}, r_{3}, r_{2}, r_{1} \mid q_{2}, q_{1}\right)=K_{2 \rightarrow 3}\left(r_{4}, r_{3}+r_{2}, r_{1} q_{2}, q_{1}\right) .
$$


Indeed denote $p_{1}=p+r_{2}$ and $p_{3}=-p+r_{3}$ then we have the product in $(22)$

$$
\begin{aligned}
\left(\frac{p_{1}}{p_{1}^{2}}-\frac{q_{1}}{q_{1}^{2}}\right)\left(\frac{p_{3}}{p_{3}^{2}}-\frac{q_{2}}{q_{2}^{2}}\right) & \\
= & \frac{p_{1} p_{3}}{p_{1}^{2} p_{3}^{2}}+\frac{q_{1} q_{2}}{q_{1}^{2} q_{2}^{2}}-\frac{p_{1} q_{2}}{p_{1}^{2} q_{2}^{2}}-\frac{p_{3} q_{1}}{p_{3}^{2} q_{1}^{2}} \\
= & \frac{1}{2 p_{1} p_{3}}\left(\left(p_{1}+p_{3}\right)^{2}-p_{1}^{2}-p_{3}^{2}\right) \\
& +\frac{1}{2 q_{1}^{2} q_{2}^{2}}\left(\left(q_{1}+q_{2}\right)^{2}-q_{1}^{2}-q_{2}^{2}\right) \\
& \left.-\frac{1}{2 p_{1}^{2} q_{2}^{2}}\left(\left(p_{1}+q_{2}\right)^{2}\right)-p_{1}^{2}-q_{2}^{2}\right) \\
& -\frac{1}{2 p_{3}^{2} q_{1}^{2}}\left(\left(p_{3}+q_{1}\right)^{2}-p_{3}^{2}-q_{1}^{2}\right) \\
= & \frac{\left(p_{1}+p_{3}\right)^{2}}{2 p_{1}^{2} p_{3}^{2}}+\frac{\left(q_{1}+q_{2}\right)^{2}}{2 q_{1}^{2} q_{2}^{2}} \\
& -\frac{\left(p_{1}+q_{2}\right)^{2}}{2 p_{1}^{2} q_{2}^{2}}-\frac{\left.p_{3}+q_{1}\right)^{2}}{2 p_{3}^{2} q_{1}^{2}} \\
= & \frac{1}{2} K_{2 \rightarrow 4}\left(r_{4}, r_{3}+r_{2}, r_{1} \mid q_{2}, q_{1}\right) .
\end{aligned}
$$

Here all vectors are here Euclidean 2-dimensional. In the last expression in (24) we take into account that $p_{1}+p_{3}=$ $r_{2}+r_{3}$

So we finally find

$$
\begin{aligned}
H_{2}(e)= & \left.\frac{1}{4\left|e_{-}\right|} g^{4} N^{4} \int d \tau_{\perp} D^{(0)}\left(q_{1}, q_{2}\right) P^{(0)} r_{1}, r_{2}\right) P^{(0)} \\
& \left(r_{4} r_{3}\right) K_{2 \rightarrow 4}\left(r_{4}, r_{3}, r_{2}, r_{1} \mid q_{2}, q_{1}\right)
\end{aligned}
$$

with $q_{1}+q_{2}=0$ and $r_{1}+r_{2}=-r_{3}-r_{4}=e$. This is the same expression which one obtains using the dispersive multiple cut approach and passing to the transverse space, except for the new factor $1 /\left|e_{-}\right|$.

\section{Three reggeons attached to the projectile}

Four diagrams corresponding to this amplitude are shown in Fig. 6. For the diffractive contribution the cut should go in between the two targets.

In the lowest order of perturbations the blobs can be taken as $q \bar{q}$ loops with three reggeon attached to the quarks and antiquarks in all different ways in the projectile and two reggeons in each target. Apart from the transverse momenta the projectile impact factor depends on $q_{1-}$ and $q_{2-}$ with $q_{3-}=-q_{1-}-q_{2-}$. The target impact factors depend on " + " components of $r$. The central blob is the vertex $\Gamma_{3 R \rightarrow 4 R}$ for the transition of the three incoming reggeons into the 4 outgoing ones with one of the reggeons not participating in the interaction. Apart from the transverse momenta

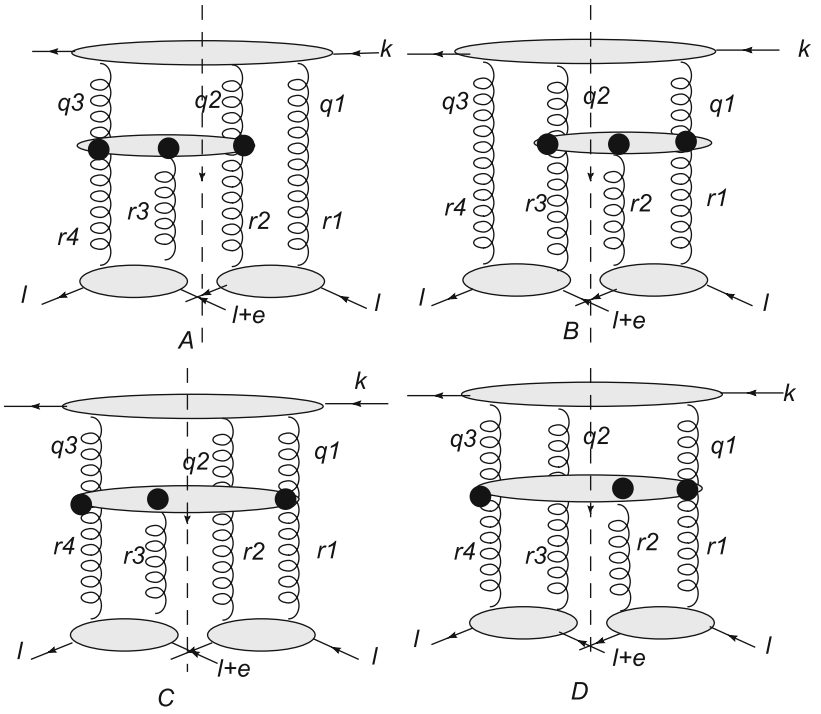

Fig. 6 The diffractive amplitudes with three reggeons attached to the projectile

it depends on $q_{1+}, q_{2+}, r_{1-}$ and $r_{4-}$. As before one has $r_{1}+r_{2}=-r_{3}-r_{4}=e$ with $e_{+}=0$.

As before integrations over $q_{1-}, q_{2-} r_{1+}$ and $r_{4+}$ are factored out. Only the impact factors depend on them. So one can immediately do these integrations by using energetic variables $\left(k-q_{1}\right)^{2},\left(k-q_{1}-q_{2}\right)^{2},\left(l+r_{1}\right)^{2}$ and $\left(l+r_{4}\right)^{2}$ and rotating the Feynman integration contour around the cut of the impact factors. In this way one obtains the standard impact functions $D_{3}^{(0)}$ for the projectile (see e.g. [9]) and $D^{(0)}$ for the targets depending only on the corresponding transverse momenta of the attached reggeons, multiplied by the overall factor $2 s^{2} / k_{+}$entering (3). Also since the singularities of the impact factors are supposed to lie at limited values the momenta, $q_{-}, r_{1+}$ and $r_{4+}$ become zero at large energies. As a result the incoming reggeons acquire $q_{-}=0$ and the outgoing ones $r_{+}=0$ in accordance with the effective action. Note that for the reggeon which does not interact one has $q_{+}=r_{-}=0$. In this way one obtains the diffractive amplitude $\mathrm{H}_{3}$ coming from the contributions with three reggeons attached to the projectile as a sum of integrals

$$
\begin{aligned}
& H_{3}^{A, B, C . D}(e)=\int d \tau_{\perp} D_{3}^{(0)}\left(q_{3}, q_{2}, q_{1}\right) P^{(0)} \\
& \quad\left(r_{1}, r_{2}\right) P^{(0)}\left(r_{4}, r_{3}\right) \int \frac{d q_{+} d r_{-}}{(2 \pi)^{2}} \Gamma_{2 R \rightarrow 4 R}^{A, B, C, D}\left(q_{+}, r_{-}\right),
\end{aligned}
$$

where the integration variables $q_{+}$and $r_{-}$refer to different momenta in the diagrams A,...D corresponding to two remaining integrations over longitudinal momenta.

The integration over $q_{3+}$ in Fig. 6a and $\mathrm{c}$ is done due to the cut which provides $-2 \pi \delta\left(\left(q_{3}+e\right)^{2}\right)$. One gets factor $-1 / 2\left|e_{-}\right|$and puts $q_{3+}=-p_{\perp}^{2} / 2 e_{-}<0$. The integration over $q_{1+}$ in Fig. $6 \mathrm{~b}, \mathrm{~d}$ is done using $-2 \pi \delta\left(\left(q_{a}-e\right)^{2}\right)$. One 
gets the same factor $-1 / 2\left|e_{-}\right|$and puts $q_{1+}=p_{\perp}^{2} / 2 e_{-}>0$. Integrations over $r_{4}$ in diagrams of Fig. 5a, c give $-1 / 4\left|q_{3+}\right|$ and that over $r_{1-}$ in diagrams $\mathrm{B}$ and $\mathrm{D}$ give factor $-1 /\left|q_{1+}\right|$.

The rest part of $\Gamma_{3 R \rightarrow 4 R}\left(q_{1+}, r_{1-}, r_{2-}\right)$ in diagrams in Fig. 5 contains products of vertices $\Gamma_{R \rightarrow G+R}$ and integrated $\Gamma_{R \rightarrow G+2 R}$ namely

$$
\begin{aligned}
H_{3}^{A} & =\frac{1}{2\left|e_{-}\right|\left|q_{3+}\right|} g^{4} C_{A} \int d \tau_{\perp} D_{3}^{(0)}\left(q_{3}, q_{2}, r_{1}\right) \\
& \times P^{(0)}\left(r_{1}, r_{2}\right) P^{(0)}\left(r_{4}, r_{3}\right) q_{3+} B\left(-p, r_{3}, r_{4}\right) L\left(p, r_{2}\right), \\
H_{3}^{B} & =-\frac{1}{2\left|e_{-}\right|\left|q_{1+}\right|} g^{4} C_{B} \int d \tau_{\perp} D_{3}^{(0)}\left(r_{4}, q_{2}, q_{1}\right) \\
& \times P^{(0)}\left(r_{1}, r_{2}\right) P^{(0)}\left(r_{4}, r_{3}\right) q_{1+} B\left(p, r_{2}, r_{1}\right) L\left(-p, r_{3}\right), \\
H_{3}^{C} & =\frac{1}{2\left|e_{-}\right|\left|q_{3+}\right|} g^{4} C_{C} \int d \tau_{\perp} D_{3}^{(0)}\left(q_{3}, r_{2}, q_{1}\right) \\
& \times P^{(0)}\left(r_{1}, r_{2}\right) P^{(0)}\left(r_{4}, r_{3}\right) q_{3+} B\left(-p, r_{3}, r_{4}\right) L\left(p, r_{1}\right), \\
H_{3}^{D} & =-\frac{1}{2\left|e_{-}\right|\left|q_{3+}\right|} g^{4} C_{D} \int d \tau_{\perp} D_{3}^{(0)}\left(q_{3}, r_{2}, q_{1}\right) \\
& \times P^{(0)}\left(r_{1}, r_{2}\right) P^{(0)}\left(r_{4}, r_{3}\right) q_{1+} B\left(p, r_{2}, r_{1}\right) L\left(-p, r_{4}\right) .
\end{aligned}
$$

Here $C$ 's are the colour factors.. Note that conjugation of $q_{3} B\left(-p, 3_{3}, r_{4}\right)$ does not change it but conjugation of $L\left(-p, r_{3}\right)$ or $L\left(-p, r_{4}\right)$ changes their sign. Hence an additional minus in $H_{3}^{B}$ and $H_{3}^{D}$. Now we take into account that $q_{1+} /\left|q_{1+}\right|=-q_{3+} /\left|q_{3+}\right|=1$, so that the final numerical coefficients are all equal to -1 in all $H_{3}$ 's. We use (24) to express the product $B \otimes L$ summed over polarizations via the Bartels kernel $K_{2 \rightarrow 3}$. We get considering all vectors Euclidean 2-dimensional

$$
\begin{aligned}
& B\left(-p, r_{3}, r_{4}\right) L\left(p, r_{2}\right) \\
& =\left(\frac{-p+r_{3}}{\left(-p+r_{3}\right)^{2}}-\frac{q_{3}}{q_{3}^{2}}\right)\left(\frac{p}{p^{2}}-\frac{p+r_{2}}{\left(p+r_{2}\right)^{2}}\right) \\
& =\frac{1}{2} K_{2 \rightarrow 3}\left(r_{4}, r_{3}, r_{2} \mid q_{3}, q_{2}\right) \text {, } \\
& B\left(p, r_{2}, r_{1}\right) L\left(-p, r_{3}\right) \\
& =\left(\frac{p+r_{2}}{\left(p+r_{2}\right)^{2}}-\frac{q_{1}}{q_{1}^{2}}\right)\left(-\frac{p}{p^{2}}+\frac{p-r_{3}}{\left(p-r_{2}\right)^{2}}\right) \\
& =\frac{1}{2} K_{2 \rightarrow 3}\left(r_{3}, r_{2}, r_{1} \mid q_{2}, q_{1}\right) \text {, } \\
& B\left(-p, r_{3}, r_{4}\right) L\left(p, r_{1}\right) \\
& =\left(\frac{-p+r_{3}}{\left(-p+r_{3}\right)^{2}}-\frac{q_{3}}{q_{3}^{2}}\right)\left(\frac{p}{p^{2}}-\frac{p+r_{1}}{\left(p+r_{1}\right)^{2}}\right) \\
& =\frac{1}{2} K_{2 \rightarrow 3}\left(r_{4}, r_{3}, r_{1} \mid q_{3}, q_{1}\right) \text {, } \\
& B\left(p, r_{2}, r_{1}\right) L\left(-p, r_{4}\right) \\
& =\left(\frac{p+r_{2}}{\left(p+r_{2}\right)^{2}}-\frac{q_{1}}{q_{1}^{2}}\right)\left(-\frac{p}{p^{2}}+\frac{p-r_{4}}{\left(p-r_{4}\right)^{2}}\right) \\
& =\frac{1}{2} K_{2 \rightarrow 3}\left(r_{4}, r_{2}, r_{1} \mid q_{3}, q_{1}\right) \text {. }
\end{aligned}
$$

Now the color factors. The color factor coming from the projectile impact factor is $(-1 / 2) f^{a_{3} a_{2} a_{1}}$ [9]. So we get

$$
\begin{aligned}
& -2 C_{A}=f^{a_{3} d b_{3}} f^{d c b_{3}} f^{a_{2} a_{1} c} f^{a_{3} a_{2} a_{1}}=-N^{4}, \\
& -2 C_{C}=f^{a_{3} d b_{3}} f^{d c b_{3}} f^{a_{1} a_{2} c} f^{a_{3} a_{2} a_{1}}=N^{4}, \\
& -2 C_{B}=f^{a_{1} b_{1} d} f^{d b_{1} c} f^{a_{2} c a_{3}} f^{a_{3} a_{2} a_{1}}=-N^{4}, \\
& -2 C_{D}=f^{a_{1} b_{1} d} f^{d b_{1} c} f^{a_{3} c a_{2}} f^{a_{3} a_{2} a_{1}}=+N^{4} .
\end{aligned}
$$

Collecting all factors we finally find

$H_{3}=H_{3}^{A}+H_{3}^{B}+H_{3}^{C}+H_{3}^{D}$

where

$$
\begin{aligned}
H_{3}^{A}= & \frac{1}{8\left|e_{-}\right|} g^{4} N^{4} \int d \tau_{\perp} D_{3}^{(0)}\left(q_{3}, q_{2}, r_{1}\right) P^{(0)} \\
& \left(r_{1}, r_{2}\right) P^{(0)}\left(r_{4}, r_{3}\right) K_{2 \rightarrow 3}\left(r_{4}, r_{3}, r_{2} \mid q_{3}, q_{2}\right), \\
H_{3}^{B}= & \frac{1}{8\left|e_{-}\right|} g^{4} N^{4} \int d \tau_{\perp} D_{3}^{(0)}\left(r_{4}, q_{2}, q_{1}\right) P^{(0)} \\
& \left(r_{1}, r_{2}\right) P^{(0)}\left(r_{4}, r_{3}\right) K_{2 \rightarrow 3}\left(r_{3}, r_{2}, r_{1} \mid q_{2}, q_{1}\right), \\
H_{3}^{C}=- & \frac{1}{8\left|e_{-}\right|} g^{4} N^{4} \int d \tau_{\perp} D_{3}^{(0)} \\
& \left(q_{3}, r_{2}, q_{1}\right) P^{(0)}\left(r_{1}, r_{2}\right) P^{(0)} \\
& \left(r_{4}, r_{3}\right) K_{2 \rightarrow 3}\left(r_{4}, r_{3}, r_{1} \mid q_{3}, q_{1}\right), \\
H_{3}^{D}=- & \frac{1}{8\left|e_{-}\right|} g^{4} C_{D} \int d \tau_{\perp} D_{3}^{(0)}\left(q_{3}, r_{2}, q_{1}\right) P^{(0)} \\
& \left(r_{1}, r_{2}\right) P^{(0)}\left(r_{4}, r_{3}\right) K_{2 \rightarrow 3}\left(r_{4}, r_{2}, r_{1} \mid q_{3}, q_{1}\right) .
\end{aligned}
$$

The found $H_{3}$ again differs from the one which was obtained in the multiple cut approach only by factor $1 /\left|e_{-}\right|$, which carries the desired $e$-dependence.

\section{Four reggeons attached to the projectile}

With four reggeons attached to the projectile its impact factor depends on three minus components of the longitudinal momenta $q_{1-}, q_{2-}$ and $q_{3-}$ with $q_{4-}=-q_{1-}-q_{2-}-q_{3-}$. As before we use the energetic variables $\left(k-q_{1}\right)^{2},\left(k-q_{1}-q_{2}\right)^{2}$ and $\left.k-q_{1}-q_{2}-q_{3}\right)^{2}$ and rotate the integration contour to enclose the right-hand singularities. As a result we get the standard impact factor $D_{4}^{(0)}$ found in the multicut approach, which depends only on the transverse momenta [9]. Also all minus component of momenta $q_{i},=1, \ldots 4$ are put to zero. The target impact factors are considered as before all plus components of the outgoing reggeon momenta become equal to zero.

In the lowest order we have two non-interacting reggeons with all their longitudinal momenta zero. So they cannot be coupled to the same target, unless $e_{-}$is zero and contribute only to the low-mass diffraction. Thus with $e_{-} \neq 0$ we find four diagrams for the amplitude shown in Fig. 7. 


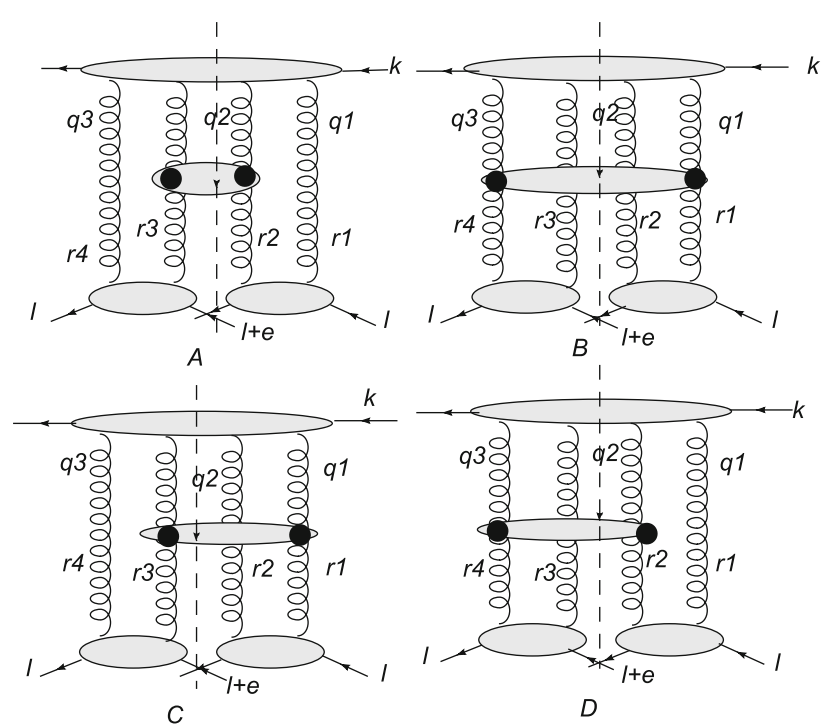

Fig. 7 The diffractive amplitudes with four reggeons attached to the projectile

The integration over $q_{2+}$ in Fig. $7 \mathrm{a}$, $\mathrm{d}$ is done due to the cut which provides $-2 \pi \delta\left(\left(q_{2}-e\right)^{2}\right)$. One gets factor $-1 / 2\left|e_{-}\right|$ and puts $q_{2+}=-p_{\perp}^{2} / 2 e_{-}<0$. The integration over $q_{1+}$ in Fig. $7 \mathrm{~b}, \mathrm{c}$ is done using $-2 \pi \delta\left(\left(q_{1}-e\right)^{2}\right)$. One gets the same factor $-1 / 2\left|e_{-}\right|$and puts $q_{1+}=p_{\perp}^{2} / 2 e_{-}>0$. There are no additional longitudinal integrations.

At this point it is convenient to sum over colors. The projectile impact factor contains two pieces with different color factors [9]

$D_{5}=-g^{2} d^{a_{4} a_{3} a_{2} a_{1}} F_{1}^{(0)}--g^{2} d^{a_{3} a_{4} a_{2} a_{1}} F_{2}^{(0)}$,

where

$$
\begin{aligned}
F_{1}^{(0)}= & D^{(0)}(1,234)+D^{0}(4,123)-D^{(0)}(14,23) \\
F_{2}^{(0)}= & D^{(0)}(124,3)+D^{(0)}(134,2) \\
& -D^{(0)}(12,34)-D^{(0)}(13,24) .
\end{aligned}
$$

Here

$d^{a b c d}=\operatorname{Tr}\left(t^{a} t^{b} t^{c} t^{d}+t^{d} t^{c} t^{b} t^{a}\right)$

and we denote $q_{1}=1, q_{1}+q_{2}=12$ etc. Summation over colors gives

$$
\begin{aligned}
& \frac{1}{4} g^{2} N^{4}\left(F_{1}^{(0)}-F_{2}^{(0)}\right) \text { for } A, B, \\
& -\frac{1}{4} g^{2} N^{4}\left(F_{1}^{(0)}-F_{2}^{(0)}\right) \text { for } C, D .
\end{aligned}
$$

So we find the amplitude as the transversal integral over the product of two Lipatov vertices together with the impact factors. Namely

$$
\begin{aligned}
H_{4}^{A}=- & \frac{1}{8\left|e_{-}\right|} g^{4} N^{4} \int d \tau_{\perp} F^{(0)}\left(r_{4}, q_{3}, q_{2}, r_{1}\right) \\
& L\left(-p, r_{3}\right) L\left(p, r_{2}\right) P^{(0)}\left(r_{4}, r_{3}\right) P^{(0)}\left(r_{2}, r_{1}\right), \\
H_{4}^{B}=-\frac{1}{8\left|e_{-}\right|} g^{4} N^{4} \int d \tau_{\perp} F^{(0)}\left(q_{4}, r_{3}, r_{2}, q_{1}\right) & \\
& L\left(-p, r_{4}\right) L\left(p, r_{1}\right) P^{(0)}\left(r_{4}, r_{3}\right) P^{(0)}\left(r_{2}, r_{1}\right), \\
H_{4}^{C}= & \frac{1}{8\left|e_{-}\right|} g^{4} N^{4} \int d \tau_{\perp} F^{(0)}\left(r_{4}, q_{3}, r_{2}, q_{1}\right) \\
& L\left(-p, r_{3}\right) L\left(p, r_{1}\right) P^{(0)}\left(r_{4}, r_{3}\right) P^{(0)}\left(r_{2}, r_{1}\right), \\
H_{4}^{D}= & \frac{1}{8\left|e_{-}\right|} g^{4} N^{4} \int d \tau_{\perp} F^{(0)}\left(q_{4}, r_{3}, q_{2}, r_{1}\right) \\
& L\left(-p, r_{3}\right) L\left(p, r_{2}\right) P^{(0)}\left(r_{4}, r_{3}\right) P^{(0)}\left(r_{2}, r_{1}\right) .
\end{aligned}
$$

Here

$$
F^{(0)}=F_{1}^{(0)}-F_{2}^{(0)}
$$

. The sign takes into account that conjugation changes the sign of the Lipatov vertex.

Summation over polarization leads to the BFKL kernel $K_{2 \rightarrow 2}$. We find (with Euclidean vectors) for diagram in Fig. $6 \mathrm{a}$.

$$
\begin{aligned}
& L(\left.-p, r_{3}\right) L\left(p, r_{2}\right)=\left(\frac{-p}{p^{2}}\right. \\
&\left.-\frac{-p+r_{3}}{\left(-p+r_{3}\right)^{2}}\right)\left(\frac{p}{p^{2}}-\frac{p+r_{2}}{\left.p+r_{2}\right)^{2}}\right) \\
&=-\frac{1}{p^{2}}-\frac{\left(p,-p+r_{3}\right)}{p^{2}\left(-p+r_{3}\right)^{2}} \\
&+\frac{\left(p, p+r_{2}\right)}{p^{2}\left(p+r_{3}=2\right)^{2}}+\frac{\left(-p+r_{3}, p+r_{2}\right)}{\left(-p+r_{3}\right)^{2}\left(p+r_{2}\right)^{2}} \\
&=-\frac{1}{p^{2}}-\frac{1}{2 p^{2}\left(-p+r_{3}\right)^{2}}\left(r_{3}^{2}-p^{2}-\left(-p+r_{3}\right)^{2}\right) \\
&-\frac{1}{2 p^{2}\left(p+r_{2}\right)^{2}}\left(r_{2}^{2}-p^{2}-\left(p+r_{2}\right)^{2}\right) \\
&+\frac{1}{2\left(-p+r_{3}\right)^{2}\left(p+r_{2}\right)^{2}} \\
&(\left.\left(r_{3}+r_{2}\right)^{2}-\left(-p+r_{3}\right)^{2}-\left(p+r_{2}\right)^{2}\right) \\
&= \frac{\left(r_{2}+r_{3}\right)^{2}}{\left.2\left(-p+r_{3}\right)^{2}\right)\left(p+r_{2}\right)^{2}} \\
&-\frac{r_{3}^{2}}{2 p^{2}\left(-p+r_{3}\right)^{2}}-\frac{r_{2}^{2}}{p^{2}\left(p+r_{2}\right)^{2}} \\
&= \frac{1}{2}\left(\frac{\left(r_{2}+r_{3}\right)^{2}}{q_{2}^{2} q_{3}^{2}}-\frac{r_{3}^{2}}{q_{3}^{2} p^{2}}-\frac{r_{2}^{2}}{q_{2}^{2} p^{2}}\right) \\
&= \frac{1}{2} K_{2 \rightarrow 2}\left(r_{3}, r_{2} \mid q_{3}, q_{2}\right) . \\
&
\end{aligned}
$$

Using the same results for the rest of diagrams we finally find

$$
\begin{aligned}
H_{4}^{A}=- & \frac{1}{16\left|e_{-}\right|} g^{4} N^{4} \int d \tau_{\perp} F^{(0)}\left(r_{4}, q_{3}, q_{2}, r_{1}\right) K_{2 \rightarrow 2} \\
& \left(r_{3}, r_{2} \mid q_{3}, q_{2}\right) P^{(0)}\left(r_{4}, r_{3}\right) P^{(0)}\left(r_{2}, r_{1}\right),
\end{aligned}
$$




$$
\begin{aligned}
H_{4}^{B}=- & \frac{1}{16\left|e_{-}\right|} g^{4} N^{4} \int d \tau_{\perp} F^{(0)}\left(q_{4}, r_{3}, r_{2}, q_{1}\right) K_{2 \rightarrow 2} \\
& \left(r_{4}, r_{1} \mid q_{4}, q_{1}\right) P^{(0)}\left(r_{4}, r_{3}\right) P^{(0)}\left(r_{2}, r_{1}\right), \\
H_{4}^{C}= & \frac{1}{16\left|e_{-}\right|} g^{4} N^{4} \int d \tau_{\perp} F\left({ }^{(0)} r_{4}, q_{3}, r_{2}, q_{1}\right) K_{2 \rightarrow 2} \\
& \left(r_{3}, r_{1} \mid q_{3}, q_{1}\right) P^{(0)}\left(r_{4}, r_{3}\right) P^{(0)}\left(r_{2}, r_{1}\right), \\
H_{4}^{D}= & \frac{1}{16\left|e_{-}\right|} g^{4} N^{4} \int d \tau_{\perp} F^{(0)}\left(q_{4}, r_{3}, q_{2}, r_{1}\right) K_{2 \rightarrow 2} \\
& \left(r_{4}, r_{2} \mid q_{4}, q_{2}\right) P^{(0)}\left(r_{4}, r_{3}\right) P^{(0)}\left(r_{2}, r_{1}\right) .
\end{aligned}
$$

Again we observe that the result obtained in the effective action technique differs from the one in the multicut approach only by the factor $1 /\left|e_{-}\right|$which exhibits the dependence on the longitudinal momentum transfer and is missing in the multicut technique.

\section{Evolution, triple pomeron vertices and cross-sections}

\subsection{Evolution diffractive vertex}

As we obtained in the effective action approach one obtains the same triple pomeron amplitude as derived in the multicut technique but with an extra factor $1 /\left|e_{-}\right|$, which carries the desired dependence on the longitudinal momentum transfer. The transverse integral is the same as obtained long ago [1, 2]. As a result to study the low- $x$ evolution and express the amplitude via the standard triple pomeron vertex we can use these old papers for manipulations in the transverse space (see also later papers $[19,20]$ where these manipulations are closer to the present ones).

First of all one notes that in our formulas all initial impact factors in the end are either $D^{(0)}$ as in $H_{2}$ or its combinations depending on different momenta $q_{+}$or $r_{-}$of the reggeons. This allows to rewrite our results in the form in which the integral starts with $D^{(0)}$ integrated over its arguments. We get for the whole amplitude (in Euclidean 2 dimensional momenta)

$$
\begin{aligned}
H^{(0)}= & \frac{1}{\left|e_{-}\right|} \int d \tau D^{(0)}(q,-q) P^{(0)}\left(r_{1}, e-r_{1}\right) P^{(0)} \\
& \left(r_{4},-e-r_{4}\right) Z\left(r_{4}, r_{1} \mid q\right),
\end{aligned}
$$

where $d \tau=d^{2} q d^{2} r_{1} d^{2} r_{4}(2 \pi)^{-9}$ and the so called diffractive triple pomeron vertex $Z\left(r_{4}, r_{1} \mid q\right)$ [19,21] corresponds to the sum of all transitions from 2,3 and 4 initial reggeons as obtained after passing to integration to the "+" momentum in all $D^{(0)}$ in the projectile impact factors.

This expression corresponds to the lowest order in the coupling constant. In the leading log approximation higher orders correspond to introducing either BFKL interactions between the reggeons coupled to the same $D^{(0)}$ or Regge trajectories into the reggeon propagators. They describe low $x$ evolution. At this point we can use our old result that this evolution leads to the change of all three $D^{(0)}$ in (33) into the fully evolved $D_{y}$ which are obtained after evolution in rapidity up to $y$ according to BFKL equation. The standard pomeron at rapidity $y$ is just $D_{y}\left(k_{1}, k_{2}\right) / k_{1}^{2} k_{2}^{2}$. The rapidity $y$ is measured by its value for the real gluon in the cut

$y=\frac{1}{2} \ln \frac{p_{+}}{p_{-}}=\frac{1}{2} \ln \frac{p^{2}}{2\left|e_{-}\right|^{2}}=\ln \frac{p}{\left|e_{-}\right| \sqrt{2}}$.

Momentum $p$ is in principle determined by the integration variable in (33). In the contribution $\mathrm{H}_{2}$ with only two reggeons coupled to the projectile we find $p^{2}=\left(q-r_{1}\right)^{2}$. However with more reggeons coupled to the projectile this relation is more complicated due to the passage to the arguments of $D^{(0)}$ in $D_{3}^{(0)}$ or $D_{4}^{(0)}$. So rigorously speaking rapidity $y$ is different for different contributions to our amplitudes. However our derivation is valid for large values of $y$ and the average $p$ is determined by the transverse dimension $R$ of the participant particles which is finite. So in fact with a logarithmic precision $y=-\ln \left|e_{-}\right|$and can be considered the same for all contributions.

So after evolution at large rapidities neglecting their variation with $p^{2}$ at fixed rapidity $y$ we get the amplitude $H^{(H M)}$ corresponding to high-mass diffraction

$$
\begin{aligned}
H^{(H M)}\left(e_{-}\right)= & \frac{1}{\left|e_{-}\right|} \int d \tau D_{Y-y}(q,-q) P_{y} \\
& \left(r_{1}, e-r_{1}\right) P_{y}\left(r_{4},-e-r_{4}\right) Z\left(r_{4}, r_{1} \mid q\right),
\end{aligned}
$$

where $y=-\ln \left|e_{-}\right|$and $Y$ is the overall rapidity of the collision. Remarkably evolution only changes the incoming and out going pomerons, the diffractive vertex $Z$, which describes their interaction, remains intact. Inserting this expression into (4) we find the cross-section for high-mass diffractive crosssection

$$
\begin{aligned}
d \sigma= & \frac{d M^{2} d^{2} l_{\perp}^{\prime}}{8 \pi^{3} M^{2}} \int d \tau D_{Y-y} \\
& (q,-q) P_{y}\left(r_{1}, e-r_{1}\right) P_{y}\left(r_{4},-e-r_{4}\right) Z\left(r_{4}, r_{1} \mid q\right) .
\end{aligned}
$$

This is the same cross-section that was obtained in [1] long ago. So the only achievement by using the effective action is a somewhat more rigorous separation of the amplitude into the interacting pomerons and the vertex describing their interaction, which was in fact assumed to be valid in [1].

\subsection{Screening correction to the scattering on the deuteron}

Passing to the double cross-section on the composite target we first note that after evolution not only diagrams similar to the high-mass diffraction, Fig. 8a, contribute to the cross-section but also ones with actually zero-mass diffraction described by the double pomeron exchange Fig. 8b, 


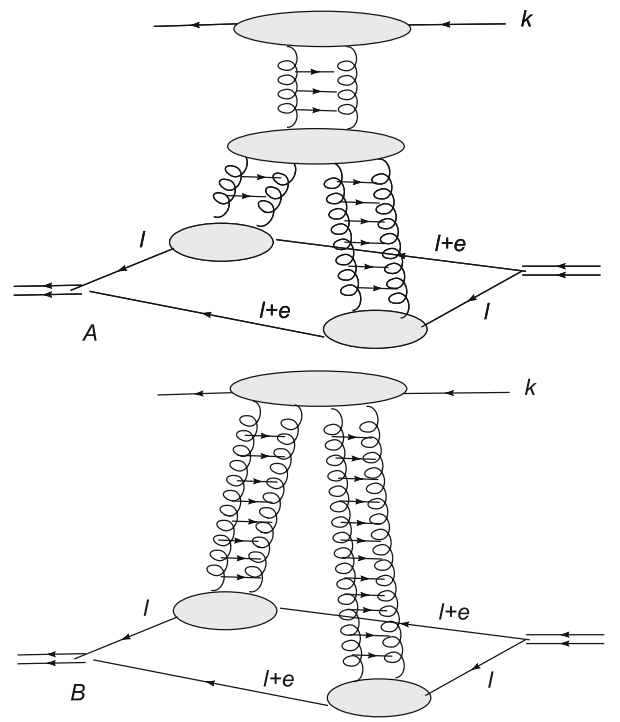

Fig. 8 High-mass diffraction (a) and double pomeron exchange (b)

omitted up to now. So we have to add the double pomeron exchange (DP) contribution. Unlike our previous calculations for this contribution energy $\left(k-q_{1}-q_{2}\right)^{2}=(k-e)^{2}$ (in Lorenz vectors) is fixed. To get the standard impact factor one should integrate over $e_{-}$Actually according to our expressions for the double scattering (9) or (10) we need precisely the integral of the $H^{(D P)}$ over $e_{-}$. Taking as $(k-e)^{2}$ in 4-dimensional Lorenz momenta as variable and closing the contour on the discontinuity of the projectile blob we obtain the impact parameter $D_{5}^{(0)}$ coupled to two outgoing pomerons. So we find the DP contribution to the cross-section as

$$
\int d e_{-} H^{(D P)}\left(e_{-}\right)=\int d \tau F^{(0)}\left(r_{4},-r_{4},-r_{1}, r_{1}\right) P_{Y}
$$

To find the total contribution we take into account that $d\left|e_{-}\right| /\left|e_{-}\right|=d y$ so that the contribution from the high-mass diffraction is

$$
\begin{aligned}
\int d e_{-} H^{(H M)}\left(e_{-}\right)= & \int_{0}^{Y} d y \int d \tau D_{Y-y}(q,-q) P_{y} \\
& \left(r_{1},-r_{1}\right) P_{y}\left(r_{4},-r_{4}\right) Z\left(r_{4}, r_{1} \mid q\right) .
\end{aligned}
$$

As was demonstrated long ago $[1,20,21]$ the sum of DP contribution and that from the diffractive vertex (38 can be rewritten such a way that the double pomeron exchange is eliminated and instead a single pomeron exchange appears together with the standard triple pomeron vertex $V$ :

$$
\int d e_{-}\left(H^{(D P)}+H^{(H M)}\right)=\int d e_{-}\left(H^{(R)}+H^{(3 P)}\right) .
$$

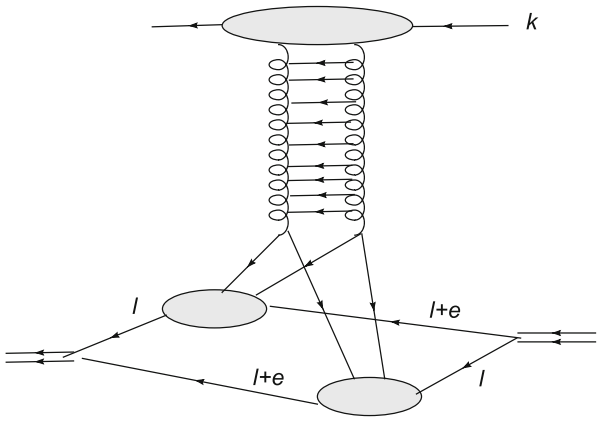

Fig. 9 The amplitude with the reggeized piece $D^{R}$

The two parts $H^{(R)}$ and $H^{(3 P)}$ behave differently at high energies: The so-called reggeized piece $H^{(R)}$ is shown in Fig. 9. Integrated over $e_{-}$it is just the impact factor $F^{(0)}$ for the four reggeons attached to the projectile in which all functions $D^{(0)}$ are substituted by their evolved expressions $D_{Y}$ with all their arguments retained. The second part $D^{(3 P)}$ is given by (38) in which the diffractive vertex $Z$ is substituted by the standard triple pomeron vertex $V$. The latter can be conveniently written in the coordinate space [20]

$\Gamma^{3 P}\left(x_{1}, x_{2}, x_{3}\right)=-\frac{g^{4} N}{4 \pi^{3}} \frac{\left(x_{1}-x_{3}\right)^{2} \nabla_{1}^{2} \nabla_{3}^{2}}{\left(x_{1}-x_{2}\right)^{2}\left(x_{2}-x_{3}\right)^{2}}$,

where each pomeron is assumed to contain factor $N$.

Using (39) we find the cross-section for the double scattering on the deuteron as a sum of two terms

$\sigma_{d}^{\text {double }}=\sigma_{d}^{(R)}+\sigma_{d}^{(3 P)}$,

where

$$
\begin{aligned}
\sigma_{d}^{(R)}=- & \frac{1}{2 \pi^{3}}\left\langle\frac{1}{r^{2}}\right\rangle_{d} \int \frac{d^{2} r_{1} d^{2} r_{4}}{\left(8 \pi^{3}\right)^{2}} F_{Y}\left(r_{4},-r_{4},-r_{1}, r_{1}\right) P^{(0)} \\
& \left(r_{1},-r_{1}\right) P^{(0)}\left(-r_{4}, r_{4}\right)
\end{aligned}
$$

and

$$
\begin{aligned}
\sigma_{d}^{(3 P)}=- & \frac{1}{\pi^{2}}\left\langle\frac{1}{r^{2}}\right\rangle_{d} \int_{0}^{Y} d y \int \frac{d^{2} q d^{2} r_{1} d^{2} r_{4}}{\left(8 \pi^{3}\right)^{3}} D_{Y-y}(q,-q) P_{y} \\
& \left(r_{1},-r_{1}\right) P_{y}\left(-r_{4}, r_{4}\right) V\left(r_{4}, r_{1} \mid q\right) .
\end{aligned}
$$

Here $F_{Y}=F^{(0)}\left(D^{0} \rightarrow D_{Y}\right)$. Both $\sigma_{d}^{(R)}$ and $\sigma_{d}^{(3 P)}$ turn out to be negative. Their sum gives the so-called screening correction to the main part of the cross-section given by the sum of the cross-sections on the proton and on the neutron. In the BFKL approach the latter is just the sum of single pomeron exchanges for the proton and for the neutron. The advantage of splitting the cross-section into parts $\sigma^{(R)}$ and $\sigma^{(3 P)}$ lies in their different behavior at large energies: part $\sigma^{(R)}$ grows as a single pomeron and part $\sigma^{(3 P)}$ grows twice rapidly, as two pomerons.

The double cross-section on the nucleus is obtained from (9) in a similar manner. 
The reggeized term (42) can be simplified when we use the explicit form of $F_{y}$. From (31) we see that $F_{y}\left(r_{4},-r_{4},-r_{1}, r_{1}\right)$ contains terms which depend only on $r_{1}$, only on $r_{4}$, terms which depend neither on $r_{1}$ nor on $r_{2}$ and finally terms which depend on $r_{1} \pm r_{4}$. The first three groups do not give contribution, since according to color transparency $P_{Y}(r,-r)$ integrated over its momentum gives zero. In the coordinate space it describes the dipole of zero dimension. Note that this property is valid at all values of $Y$ and at $Y=0$ in particular. We assume that $P^{(0)}(r,-r)$ can be taken as the limit of $P_{Y}(r,-r)$ at $Y \rightarrow 0$. This follows from its expression via the Green function in which the coupling constant enters only through the combination $Y E$ where $E$ are the BFKL levels, which vanishes when $Y \rightarrow 0$ or $g \rightarrow 0$. Obviously this is a regularization of the expression for $P^{(0)}(r,-r)=D^{(0)}(r,-r) / r^{4}$. Thus the only terms in $F_{y}$ (which give the same contribution) are $-D_{Y}\left(r_{1}+r_{4},-r_{1}-r_{4}\right)$ and $-D_{Y}\left(r_{1}-r_{4},-r_{1}+r_{4}\right)$. So writing $\left.D_{Y}^{(} q,-q\right)$ simply as $D_{Y}(q)$ we get the reggeized part as

$$
\begin{array}{r}
\sigma_{d}^{(R)}=\frac{1}{2 \pi^{3}}\left\langle\frac{1}{r^{2}}\right\rangle_{d} \int \frac{d^{2} r_{1} d^{2} r_{4}}{\left(8 \pi^{3}\right)^{2}} D^{(0)} \\
\left(r_{1}\right) D^{(0)}\left(r_{4}\right) \frac{D_{Y}\left(r_{1}+r_{4}\right)}{r_{1}^{4} r_{4}^{4}} .
\end{array}
$$

In this form convergence at small $r_{1}$ and $r_{4}$ is not obvious. $D^{(0)}$ vanishes at $r \rightarrow 0$ at least as $r^{2}$ but this may lead to the logarithmic divergence. However one can subtract from $D_{Y}\left(r_{1}+r_{4}\right)$ its values at $r_{1}=0$ and $r_{3}=0$ without changing the result

$$
\begin{aligned}
\sigma_{d}^{(R)}= & \frac{1}{2 \pi^{3}}\left\langle\frac{1}{r^{2}}\right\rangle_{d} \int \frac{d^{2} r_{1} d_{2} r_{4}}{\left(8 \pi^{3}\right)^{2}} D^{(0)}\left(r_{1}\right) D^{(0)}\left(r_{4}\right) \\
& \frac{D_{Y}\left(r_{1}+r_{4}\right)-D_{Y}\left(r_{1}\right)-D_{Y}\left(r_{4}\right)}{r_{1}^{4} r_{4}^{4}}
\end{aligned}
$$

Now the numerator vanishes when either $r_{1}$ or $r_{4}$ are equal to zero, which provides convergence at $k_{1}=0$ or $k_{4}=0$. The form (45) for the reggeized part can be used for practical calculations.

The total cross-section on the deuteron is thus

$\sigma_{d}^{\text {tot }}=\sigma_{p}+\sigma_{n}-\sigma^{\text {screen }} \simeq 2 \sigma_{p}-\sigma^{\text {screen }}$,

where $\sigma^{\text {screen }}$ is the sum (41) with the opposite sign, which turns out to be positive.

It is instructive to compare the two components of the screening correction for a more or less realistic situation. Qualitative estimate show that

$\sigma_{d}^{(R)}(Y) \sim \bar{\alpha}_{s}^{2} e^{\Delta Y}, \quad \sigma_{d}^{(3 P)}(Y) \sim \bar{\alpha}_{s}^{2} e^{2 \Delta Y}$, where $\bar{\alpha}_{s}=\alpha_{s} N / \pi$ and $\Delta=4 \bar{\alpha}_{s} \ln 2$. Also both contain the small factor $<1 / r^{2}>_{d} \simeq 0.48 \mathrm{fm}^{-2}$ (with the Hulthen wave function). So with a small coupling constant and finite $Y$ both terms in the screening correction are small compared to the main part $2 \sigma_{p}$. With the growth of $Y$ the ratio $\sigma_{d}^{(R)} / 2 \sigma_{p}$ remains intact whereas the ratio $\sigma_{d}^{(3 P)} / 2 \sigma_{p}$ grows as $\exp (\Delta Y)$. So at sufficiently high $Y$ this ratio becomes greater than unity and the total cross-section becomes negative. Obviously such values of $Y$ lie outside the applicability of the BFKL approach in the leading log approximation.

To make more quantitative estimations we choose the coupling constant to have the intercept $\Delta$ more or less in accordance with the observed growth of $\sigma_{p}(Y)$ at large $Y$. We choose $\Delta=0.12$ which fixes the coupling constant to be quite small $\bar{\alpha}_{s}=0.0432$. In calculating $\sigma_{d}^{(3 P)}$ we encounter a problem with the infrared behavior. Actually we do not find any infrared divergence. However the important values of momenta at large rapidities shift deep into the infrared region making the contribution abnormally large and providing an additional growth with $Y$. So one has to impose the confinement and restrict small values of momenta to lie in the physically reasonable interval $q>q_{\min } \sim \Lambda_{Q C D}$. Taking the projectile to be the proton and perform calculations one has to choose the form of the proton color density. We take it to be

$\Phi(q)=q^{2} \rho(q), \quad \rho(q)=\gamma e^{-\beta q^{2}}$,

where $\beta$ is dictated by the proton radius $0.08 \mathrm{fm}$ and $\gamma$ is determined from the proton cross-section at small $Y$ which we take $4 \mathrm{fm}^{2}$.

Relegating some details of the calculation to Appendix we only present here our results. We assume $Y$ to be large so that one can use the asymptotic expressions for the relevant pomeron functions. As expected the ratio of the reggeized part to the main one does not depend on $Y$ and

$$
-\frac{\sigma^{(R)}(Y)}{2 \sigma_{p}(Y)}=0.00566
$$

The ratio of the triple pomeron part to the main one turns out to be still much smaller. But it steadily grows with $Y$. We present $r(Y)=-\sigma^{(3 P)}(Y) / 2 \sigma_{p}(Y)$ in Fig. 10 in the left panel.

Just for illustration we also consider the case of a much larger coupling constant $\bar{\alpha}_{s}=0.2$ frequently used in many older calculations. With this choice

$$
-\frac{\sigma^{(R)}(Y)}{2 \sigma_{p}(Y)}=0.121
$$

and ratio $r(Y)$ is shown in Fig. 10 in the right panel 

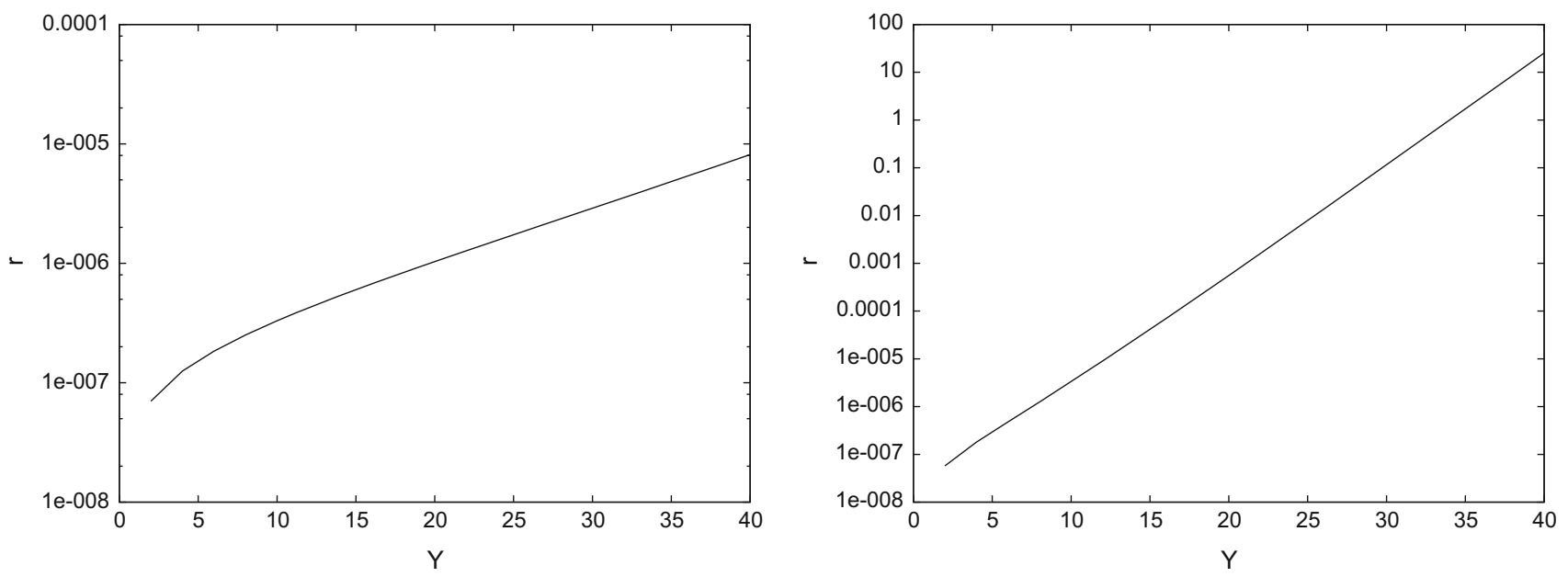

Fig. 10 The ratio of the triple pomeron part to the main one with the opposite sign with $\bar{\alpha}_{s}=0.0432$ (the left panel) and with $\bar{\alpha}_{s}=0.2$ (the right panel)

\section{Conclusions}

We have studied the triple-pomeron amplitude in the effective action formalism with the aim of deriving its dependence on the transferred longitudinal momentum, necessary for the calculation of cross-sections. We limited ourselves to the imaginary part of the amplitude, which substantially simplified our task. Our results turned out quite simple: the dependence on the longitudinal momentum transfer is separated into a simple extra factor. Using old studies performed in the multiple cut approach we transformed our amplitudes and the resulting cross-sections into the more or less standard forms where either the single or double pomeron exchange appear accompanied by normal or diffractive triple pomeron vertices respectively. The found high-mass diffractive crosssection off a hadron coincides with the known one, On the other hand our results allow to obtain a rigorous expression for the double scattering cross-sections on a composite target. Estimation indicate that at present energies the screening corrections corresponding to the double cross-section are dominated by the so-called reggeized contribution, which however is still much smaller tan the bulk given by the sum of the cross-sections on the constituents

In principle the effective action approach allows to calculate also the real part of the amplitudes. This is necessary for instance to find the elastic cross-sections off the composite target. Unfortunately such calculations turn out to be much more complicated due to the necessity to use a general gauge and struggle against appearing divergencies. We retain our hope to advance in this direction.

Data Availability Statement This manuscript has no associated data or the data will not be deposited. [Authors' comment: There is no data, since no external date has been used and those for Fig (10) are directly obtainable via Eq. (23) and (45).]
Open Access This article is licensed under a Creative Commons Attribution 4.0 International License, which permits use, sharing, adaptation, distribution and reproduction in any medium or format, as long as you give appropriate credit to the original author(s) and the source, provide a link to the Creative Commons licence, and indicate if changes were made. The images or other third party material in this article are included in the article's Creative Commons licence, unless indicated otherwise in a credit line to the material. If material is not included in the article's Creative Commons licence and your intended use is not permitted by statutory regulation or exceeds the permitted use, you will need to obtain permission directly from the copyright holder. To view a copy of this licence, visit http://creativecomm ons.org/licenses/by/4.0/.

Funded by $\mathrm{SCOAP}^{3}$.

\section{Appendix. Some details on the calculation of $\sigma_{d}^{(3 P)}$ and $\sigma_{d}^{(R)}$}

\subsection{BFKL details}

As a basis we use the leading semi-amputated (SA) eigenfunctions of the BFKL equation in the forward direction

$\phi_{n=0 v}(k, \chi)=\sqrt{2} k^{-1+2 i v},-\infty<v<+\infty$.

The full pomeron eigenfunction is $\Phi_{0 v}(k)=\phi_{0 v}(k) / k^{2}$. The corresponding SA Green function is

$g_{y}\left(k, k^{\prime}\right)=\int d \nu e^{y \omega_{0 v}} \phi_{0 v}(k) \phi_{0 v}^{*}\left(k^{\prime}\right)$,

where the gluon trajectories $\omega_{0 v}$ are the known eigenvalues of the BFKL equation. At $y=0$ we have

$g_{0}\left(k, k^{\prime}\right)=\frac{2 \pi}{k} \delta\left(k-k^{\prime}\right)$ 
and at large $y$

$g_{y}^{a s}\left(k, k^{\prime}\right)=\frac{2}{k k^{\prime}} e^{y \Delta} \sqrt{\frac{\pi}{a y}} \exp \left(-\frac{\ln ^{2}\left(k^{2} / k^{\prime 2}\right)}{4 a y}\right)$,

where $a=14 \bar{\alpha}_{s} \zeta(3)$.

For the pp scattering with the proton color density (47) we find at $y=0$

$\sigma_{p}(Y=0) \equiv \sigma_{0}=\int_{0}^{\infty} \frac{d k^{2}}{2 \pi} \rho^{2}(k)=\frac{\gamma^{2}}{4 \pi \beta}$

and at large $y$

$\sigma_{p}^{a s}=e^{y \Delta} \sqrt{\frac{\pi}{a y}}\left(\int_{0}^{\infty} \frac{d k}{2 \pi} \rho(k)\right)^{2}=\sigma_{0} e^{y \Delta} \sqrt{\frac{\pi}{a y}}$.

The SA pomeron coupled to the color density (47) at high energies is given by

$$
\begin{aligned}
& \phi^{a s}(q)=\frac{2}{q} e^{y \Delta} \sqrt{\frac{\pi}{a y}} \int \frac{d^{2} k}{(2 \pi)^{2} k} \rho(k) \\
& \times \exp \left(-\frac{\ln ^{2}\left(q^{2} / k^{2}\right)}{4 a y}\right) .
\end{aligned}
$$

\subsection{The triple pomeron contribution}

The bulk of the contribution $\sigma_{d}^{(3 P)}$ given by Eq. (43) was studied in our paper [22] devoted to the diffractive crosssection off the deuteron. There the integral over momenta was transformed to the coordinate space:

$$
\begin{aligned}
& \sigma_{d}^{(3 P)}=\frac{1}{32 \pi^{8}} g^{4} N^{4}\left\langle\frac{1}{r^{2}}\right\rangle_{d} \\
& \int_{0}^{Y} d y \int \frac{d^{2} x_{12} d^{2} x_{23}}{x_{12}^{2} x_{23}^{2} x_{31}^{2}} D_{Y-y}\left(x_{31}\right) P_{y}\left(x_{12}\right) P_{y}\left(x_{23}\right)
\end{aligned}
$$

with $x_{12}+x_{23}+x_{31}=0$. The sign takes into account that the triple pomeron vertex $V$ bears the minus sign. With the help of $\delta^{2}\left(x_{12}+x_{23}+31\right)$ the integral over coordinates in (57) transforms into

$J(y)=\int \frac{d^{2} q}{(2 \pi)^{2}} \psi_{y}^{2}(q) \chi_{Y-y}(q)$,

where

$\psi_{y}(q)=\int \frac{d^{2} x}{x^{2}} P_{y}(x) e^{i q x}, \quad \chi_{y}(q)=\nabla^{2} q^{4} \nabla^{2} \psi_{y}(q)$

Using the relation

$q^{2} \nabla^{2} \psi_{y}(q)=-q^{2} P_{y}(q)=-\phi_{y}(q)$

one finds that the coefficients in the expansion of $\psi(q)$ in $\phi_{0 n}$ are $-(1-2 i v)^{2}$ smaller than of $\phi(q)$ and those of $\chi(q)$ are $-(1+2 i v)^{2}$ larger the coefficients of $\phi(q)$. It follows that asymptotically at high $y$ we have $\psi(q)^{a s}=\chi(q)^{a s}=$ $-\phi(q)^{a s}$.
Note that in the integral $J(y)(58)$ the product of asymptotical $\psi^{2} \chi$ generates a singularity $1 / q^{3}$ at small $q$. As mentioned, this does not lead to any divergence due to the exponential factor. However this factor begins to play its role only at extremely small $q$ when $\ln (1 / q) \sim \sqrt{y}$. In fact integration over all values of $q$ leads to factor

$\sqrt{1 / b} \exp (1 / 4 b), \quad b=\frac{1}{a}\left(\frac{2}{y}+\frac{1}{Y-y}\right)$

with $a$ from Eq. (53). At high rapidities $b<<1$ and the factor behaves as $s$. This behavior in the infrared results as a combination of the behavior of the wave function and vertex at large distances. The pomeron wave function contains the so-called Coulomb tail due to the zero gluon mass. The triple gluon vertex for the same reason does not introduce any cut in the infrared. Still in the end the infrared divergence turns out to be actually cured, so that diffusion into the infrared becomes indeed stabilized and the final cross-section calculated with the asymptotical wave function (56) integrated over all values of $q$ is finite. However it is absurdly large, which is confirmed by numerical estimates. So the triple pomeron contribution turns out to be decisively dependent on the infrared region of momenta or equivalently on large distances. Any reasonable calculation therefore has to limit values of $q$ where the BFKL approach may be reasonable. We assume that this limitation should restrict $q$ to values above $\Lambda_{Q C D} \simeq 0.3 \mathrm{GeV} / \mathrm{c}$. Probably another way to cure this difficulty is to introduce the effective gluon mass.

To factorize the 8-dimensional integral $J$ and still retain the (part of) behavior of the exponential factor in (56) we substitute $\ln \left(k^{2}\right)$ in it by $\ln (1 / \beta)$ having in mind good convergence in $k$ with the typical value $k^{2} \sim 1 / \beta$. Using asymptotical expressions for $\psi$ and $\chi$ we find

$$
\begin{aligned}
\sigma_{d}^{(3 P)}= & -\bar{\alpha}_{s}^{2} N^{2} C_{1}\left\langle\frac{1}{r^{2}}\right\rangle_{d} \\
& \times \int_{0}^{Y} d y e^{\Delta(Y+y)} \frac{1}{y \sqrt{Y-y}} I_{1}(y) .
\end{aligned}
$$

where

$C_{1}=\frac{1}{(2 \pi)^{6}}\left(\frac{\gamma^{2}}{\beta a}\right)^{3 / 2}$

and

$I_{1}(y)=\int_{q_{\min }}^{\infty} \frac{d q}{q^{2}} \exp \left[-\frac{\ln ^{2}\left(q^{2} \beta\right)}{4 a}\left(\frac{2}{y}+\frac{1}{Y-y}\right)\right]$

The lower limit is $q_{\min }=1.5 \mathrm{fm}^{-1}$. The integral over $y$ is convergent at $y=0$, since $I(y)$ goes to zero at this point. It was calculated numerically. 


\subsection{The reggeized contribution}

The reggeized contribution is given by expression

$\sigma_{d}^{(R)}=\frac{1}{8 \pi^{5}}\left\langle\frac{1}{r^{2}}\right\rangle_{d} J_{R}$,

where

$J_{R}=\int \frac{d^{2} r_{1} d^{2} r_{4}}{(2 \pi)^{4}} P^{(0)}\left(r_{1}\right) P^{(0)}\left(r_{4}\right) D_{Y}\left(r_{1}+r_{4}\right)$.

From (56) we find the asymptotic of $D_{Y}(q)=q^{2} \phi(q)$. This together with the expression for the initial pomeron $P^{(0)}(q)=\rho(q) / q^{2}$ gives

$$
\begin{aligned}
J_{R}= & \frac{2}{(2 \pi)^{3}} e^{Y \Delta} \sqrt{\frac{\pi}{a Y}} \\
& \int \frac{d r_{1} d r_{2} d \phi}{r_{1} r_{4}} \rho\left(r_{1}\right) \rho\left(r_{4}\right) F \sqrt{r_{1}^{2}+r_{4}^{2}+2 r_{1} r_{4} \cos \phi} .
\end{aligned}
$$

To improve convergence we act as in (45) and subtract from the square root its values at $r_{1}=0$ and $r_{4}=0$. After that we can drop the exponential factor in the integration over $k$, which does not spoil convergence at small $r_{1}$ and $r_{4}$ and is certainly possible at very high $Y$. With $\rho(k)$ given by (47) we then find

$$
F=\frac{\gamma}{4 \pi} \gamma \sqrt{\frac{\pi}{\beta}} \text {. }
$$

So we have

$$
J_{R}=\frac{\gamma^{3}}{16 \pi^{3}} e^{Y \Delta} \sqrt{\frac{1}{a \beta Y}} I_{2},
$$

where

$$
\begin{aligned}
I_{2}= & \int \frac{d r_{1} d r_{4} d \phi}{r_{1} r_{4}} e^{-\beta\left(r_{1}^{2}+r_{4}^{2}\right)} \\
& \left(\sqrt{r_{1}^{2}+r_{4}^{2}+2 r_{1} r_{4} \cos \phi}-r_{1}-r_{4}\right) .
\end{aligned}
$$

$\sigma_{d}^{(R)}=\bar{\alpha}_{s}^{2} N^{2} C_{2}\left\langle\frac{1}{r^{2}}\right\rangle_{d} e^{Y \Delta} \sqrt{\frac{1}{Y}} I_{2}$,

where

$C_{2}=\frac{1}{4 \pi^{3}} \gamma^{3} \frac{1}{\sqrt{\beta a}}$.

The three-dimensional integral (68) was calculated numerically.

\section{References}

1. J. Bartels, Z. Phys. C 60, 471 (1993)

2. J. Bartels, M. Wuesthoff, Z. Phys. C 66, 157 (1995)

3. A.H. Mueller, Nucl. Phys. B 415, 373 (1994)

4. A.H. Mueller, Nucl. Phys. B 437, 107 (1995)

5. A.H. Mueller, B. Patel, Nucl. Phys. B 425, 471 (1994)

6. M.A. Braun, Eur. Phys. J. C 73, 2418 (2013)

7. V.A. Abramovsky, V.N. Gribov, O.V. Kancheli, Sov. J. Nucl. Phys. 18, 308 (1974)

8. J. Bartels, M. Ryskin, Z. Phys. C 76, 241 (1997)

9. J. Bartels, C. Ewerz, JHEP 9909, 026 (1999)

10. M. Hentschinski, dissertation (2009) arXiv:0908.2576 [hep-ph]

11. L.N. Lipatov, Nucl. Phys. B 452, 369 (1995)

12. L.N. Lipatov, Phys. Rep. 286, 131 (1997)

13. E.N. Antonov, I.O. Cherednikov, E.A. Kuraev, L.N. Lipatov, Nucl. Phys. B 721, 111 (2005)

14. L.N. Lipatov, Sov. J. Nucl. Phys. 23, 338 (1976)

15. E.A. Kuraev, L.N. Lipatov, V.S. fadin, Sov. Phys. JETP 45 (1977) 199

16. I.I. Balitsky, L.N. Lipatov, Sov. J. Nucl. Phys. 28, 822 (1978)

17. M.A. Braun, M.I. Vyazovsky, Eur. Phys. J. C 51, 103 (2007)

18. M.A. Braun, L.N. Lipatov, MYu. Salykin, M.I. Vyazovsky, Eur. Phys. J C 71, 1639 (2011)

19. M. Braun, Eur. Phys. J. C 6, 321 (1999)

20. M.A. Braun, G.P. Vacca, Eur. Phys. J. C 6, 147 (1997)

21. J. Bartels, M. Braun, G.P. Vacca, Eur. Phys. J. 40, 419 (2005)

22. M.A. Braun, Eur. phys. J. 77(5), 279 (2017)

Finally we obtain 